\title{
Information, ambiguity and price equilibrium
}

\author{
R. R. Routledge ${ }^{1}$
}

Received: 15 August 2016 / Accepted: 17 February 2017 / Published online: 28 February 2017 (C) The Author(s) 2017. This article is published with open access at Springerlink.com

\begin{abstract}
This work analyzes a price choice game with discontinuous payoffs in which sellers may have asymmetric information about the state of the market. The prices which sellers post in the market must be measurable with respect to their private information and sellers are assumed to have ex ante maximin expected utilities. Easily verified conditions upon the primitives which guarantee the existence of a pure strategy price equilibrium in the game, under both equal sharing and winner-takes-all sharing at price ties, are presented. The existence proofs are direct and constructive.
\end{abstract}

Keywords Asymmetric information - Discontinuous payoffs · Ambiguity · Maximin utilities $\cdot$ Price equilibrium $\cdot$ Ex ante payoffs

JEL Classification $\mathrm{C} 71 \cdot \mathrm{C} 73$

\section{Introduction}

A persistent foundational problem in economic theory is the explanation of how prices are formed. In most Walrasian models, it is assumed that there is an individual outside the model, the "auctioneer", who establishes the market prices and prevents trading at disequilibrium prices. An attempt to remedy this foundational problem comes from the

\footnotetext{
The author would like to thank a referee for a detailed set of comments which improved the paper. Thanks are also due to the attendees of the Liverpool economic research seminar and colleagues at the University of Liverpool, especially Christian Bach, Dominique Demougin, Yiquan Gu, Eunyoung Moon, Harvey Upton and Yunchou Wu for their encouragement and comments during the writing of this paper.
}

$凶 \quad$ R. R. Routledge

R.R.Routledge@liverpool.ac.uk

1 University of Liverpool, Liverpool, UK 
literature on price competition, the class of Bertrand and Bertrand-Edgeworth games, in which sellers choose which prices to post in the market. This research has provided important insights into the conditions under which the competitive equilibrium can be achieved as the Nash equilibrium outcome of an appropriate price game.

A general theory of price competition would include the possibility that sellers may have asymmetric information when posting prices in the market place. However, little work has been done in this direction. ${ }^{1}$ One of the reasons why little research has been done in this direction is that homogeneous-good price games have discontinuous payoffs and there are few existence results for discontinuous games with asymmetric information. Only recently, He and Yannelis (2015b, 2016) have demonstrated the existence of Nash equilibria in particular discontinuous games with asymmetric information by generalizing the existence results of Reny (1999). ${ }^{2}$

This work presents a theory of price competition under asymmetric information of the type usually studied in the general equilibrium context. In the model, sellers post prices for a single perfectly homogeneous good, and both the market demand and costs functions are state contingent. Sellers have private information which is modelled by a partition of the finite set of states of the world. Given these information restrictions, the prices which sellers post in the market must be measurable with respect to their private information. At the ex ante stage, the sellers are assumed to have maximin expected utilities (MEU) consistent with ambiguity aversion. It is demonstrated that if two conditions are satisfied (i) the sellers have symmetric cost functions (ii) there are at least two sellers of each information type, then the game possesses a pure strategy price equilibrium under both equal-sharing and winner-takes-all sharing at price ties. In Sect. 3.1, it is shown that if any of these conditions are relaxed, then a pure strategy price equilibrium may fail to exist. Therefore, it does not appear possible to relax these conditions any further.

There is both an economic motivation, and a technical requirement, behind the two conditions. The first condition, that there be at least two sellers of the same information type, is equivalent to no seller having a monopoly over their information in the market. It is standard in many economic models to consider the case where the set of sellers is replicated so no seller is a monopolist. ${ }^{3}$ If one were to fix a price game, then for any replication of the set of sellers, this condition would be satisfied. The technical requirement which having at least two sellers of each information type meets, is that it solves the well-known "open-set" problem in which a seller's best response may not be well defined. ${ }^{4}$ When there are at least two sellers of each information type, it is possible to construct equilibria in which sellers of the same type post the same

\footnotetext{
1 A step in this direction was taken by Spulber (1995) and Wambach (1999). These works studied prices games in which sellers had uncertain costs. However, both of these papers assume that the uncertainty is symmetric amongst the sellers, and they look for symmetric Nash equilibria.

2 Unfortunately, these recent existence results are not easy to apply to the model here because Bertrand games tend not to be payoff secure as tied payoffs can be greater than non-tied payoffs. Interestingly, the discussion in Reny (1999) regarding Bertrand competition under constant and symmetric marginal costs, is one of the few special cases in which the Bertrand price game is payoff secure and quasiconcave.

3 Debreu and Scarf (1963) were the first to consider markets in which the set of sellers is replicated.

4 See Vives (1999, p.123) and the discussion of footnote 8 on page 368 for details of the open-set problem.
} 
prices in the market. Therefore, each seller knows they will tie with at least one other seller, and they cannot benefit from increasing their price. The second condition, that sellers have symmetric cost functions, means that sellers have access to a common production technology which generates their symmetric cost functions. As the paper imposes convexity on the cost function, the reader can imagine that the sellers have access to a common concave production function which generates their convex cost function. The technical requirement which this condition meets, is that it ensures that the ex post games possess a pure strategy Nash equilibrium. If sellers have asymmetric costs, then even without asymmetric information being introduced into the model, a pure strategy Nash equilibrium may fail to exist. ${ }^{5}$

In this work, it is assumed that sellers are maximin expected utility (MEU) maximizers. Although this is a widely used model of choice under ambiguity, one might reasonably ask whether the results would hold if one were to adopt standard Bayesian expected utilities (BEU) instead. This is an interesting, but technically difficult, open question. As was noted earlier, existence results for discontinuous games, in which players' strategies must be measurable with respect to their private information, and players have Bayesian expected utilities, have only recently been provided by $\mathrm{He}$ and Yannelis (2015b, 2016). However, these general results are not easily applicable to the model studied here because the price game can fail to be payoff secure. Even if there were no asymmetric information in the model, the one-shot Bertrand game of the type studied in Dastidar (1995), and generalized here, is not payoff secure. Instead, existence results have tended to be obtained by directly examining the primitives of the model. With asymmetric information, however, this is not easy to do. This explains why there has been an absence of research in this direction. There is a technical advantage which MEU has over standard Bayesian utilities which this work has been able to exploit. In price games, it is necessary to stop sellers wanting to undercut their rivals' prices. If sellers have maximin expected utilities, and are uncertain about the state of the world, when considering undercutting they consider only the worst outcome. As a consequence, this makes it easier to find price vectors at which sellers are not willing to risk undercutting other sellers' prices.

\subsection{Related literature}

This work brings together two different areas of research in economic theory. First, there is a literature analyzing the existence of Nash equilibria in games where sellers post prices in homogeneous-good markets. Second, there is a literature on ambiguity, when individuals may not be able to assign probabilities to relevant outcomes, and the implications of this problem for canonical economic models. ${ }^{6}$

Dastidar (1995) studied a one-shot model of price competition in a homogeneousgood market in which sellers had strictly convex costs and demand was equally shared

\footnotetext{
5 Blume (2003) gives an example where a pure strategy Nash equilibrium fails to exist, but shows that a continuum of mixed strategy Nash equilibria do exist.

6 A detailed discussion of price competition in homogeneous-good markets can be found in Baye and Kovenock (2008). An accessible introduction to the theory of choice under uncertainty, including ambiguity and related paradoxes, such as the Newcomb problem, is Allingham (2002, Ch.3).
} 
at minimum price ties. It was proved that with symmetric cost functions there exists a continuum of pure strategy price equilibria. When the sellers have asymmetric costs, a pure strategy Nash equilibrium exists, but may be unique. Baye and Morgan (2002) considered price competition in a model with winner-takes-all sharing at minimum price ties. With this sharing rule at minimum price ties, the existence of a pure Nash equilibrium is guaranteed under weak conditions on the market primitives, including possible discontinuities in the monopoly profit function. Hoernig (2007) studied the general properties of sharing rules at minimum price ties which guarantee the existence of pure and mixed Nash equilibria. By applying an existence result of Reny (1999) to the price game, it was proved that if the sharing rule is coalition monotone and weakly tie-decreasing, the non-tied payoffs are continuous and the sum of the payoffs upper semicontinuous, then the game possesses a pure strategy Nash equilibrium. Bagh (2010) considered a model of price competition with possibly asymmetric convex costs, discontinuous demand, and an arbitrary but deterministic sharing rule under prices ties. Conditions upon the primitives which guaranteed the existence of a continuum of pure strategy Nash equilibria were presented. Saporiti and Coloma (2010) studied a model with symmetric sellers who had convex variable costs, and a possibly avoidable fixed cost of production. They established necessary and sufficient conditions for the existence of a pure strategy Nash equilibrium based upon the property of subadditivity of the cost function. Dastidar (2011) also examined the implications of subadditive symmetric cost functions for the existence of pure and mixed Nash equilibria. This work confirmed the suspected non-existence of both pure and mixed strategy Nash equilibria when sellers have symmetric increasing returns to scale cost functions.

The literature on ambiguity in economic models began with Ellsberg's (1961) famous urn experiments which demonstrated that, when individuals cannot assign probabilities to particular outcomes, then their choices may be inconsistent with standard subjected expected utility theory. Gilboa and Schmeidler (1989) proposed a theory of choice under ambiguity which can explain the observations in the Ellsberg experiments, and is analytically tractable enough to be applied to a wide variety of contexts. The model of maximin expected utility assumes that individuals evaluate ambiguous outcomes by considering the minimum utility across the set of probabilities which agree with their information. In recent years, the implications of this model have been explored in some detail. Correia-da-Silva and Hervés-Beloso (2009) analyzed an Arrow-Debreu economy with private information in which individuals chose from a list of bundles of goods. By choosing from a list of bundles, individual consumption did not need to be measurable with respect to each individual's information. When individuals were unsure which bundle of goods would be delivered, it was assumed that they considered the minimum possible utility. In de Castro and Yannelis (2011) the model of maximin expected utility was applied to asymmetric information economies and mechanism design scenarios. ${ }^{7}$ They emphasized that one of the advantages of the maximin expected utility model is that it solves the usual conflict between ex ante efficient allocations and ex post incentive compatibility. In essence, with maximin

\footnotetext{
7 They also presented an excellent discussion of the relevance and shortcomings of the maximin expected utility in Section 7.
} 
expected utilities, individuals equalize utilities across different states of the world. As a consequence, after individuals receive their private information about the state of the world, they have no incentive to misrepresent the information they have received. He and Yannelis (2015a) established equivalence theorems for the maximin expectations equilibrium and the core with an atomless measure space of individuals using maximin expected utilities to evaluate state-contingent allocations.

\section{The model}

Consider a finite set of sellers $N=\{1, \ldots, n\}, n \geq 2$, who produce a single perfectly homogeneous good. There is a finite set $\Omega=\left\{\omega_{1}, \ldots, \omega_{m}\right\}$ which is the set of states of the world, and a probability distribution, $\mu$, over the set $\Omega$. It shall be assumed that $\mu(\omega)>0$ for every $\omega \in \Omega$ so no state of the world is redundant. The state-contingent cost function of seller $i$ is $C_{i}: \Re_{+} \times \Omega \rightarrow \Re_{+}$. The following conditions are imposed upon the cost functions.

Assumption 2.1 For every $i \in N$, and every $\omega \in \Omega, C_{i}(\cdot, \omega)$ is $C^{2}$, convex, strictly increasing and satisfies $C_{i}(0, \omega)=0$.

The state-contingent market demand function for the homogeneous good is $D$ : $\Re_{+} \times \Omega \rightarrow \Re_{+}$. The following conditions are imposed upon the demand function.

Assumption 2.2 For every $\omega \in \Omega$ there exist positive finite real numbers $\bar{x}$ and $\bar{y}$ such that $D(x, \omega)=0$ for all $x \geq \bar{x}$, and $D(0, \omega)=\bar{y}$. The function $D(\cdot, \omega)$ is $C^{2}$ and strictly decreasing on $(0, \bar{x})$.

The private information of seller $i$ is given by $P_{i}$ which is a partition of the set $\Omega$. If two states of the world are in the same element in the partition $P_{i}$, then seller $i$ is unable to distinguish between those two states. A function $f: \Omega \rightarrow \Re_{+}$will be called $P_{i}$-measurable ${ }^{8}$ if, whenever $\omega_{p} \in E$ and $\omega_{q} \in E$ for some $E \in P_{i}$, then $f\left(\omega_{p}\right)=f\left(\omega_{q}\right)$. Given these information restrictions upon the sellers, the strategy set of seller $i$ is

$$
L_{i}=\left\{f: \Omega \rightarrow \Re_{+}: f \text { is } P_{i} \text {-measurable }\right\} .
$$

Let $L=\times_{i \in N} L_{i}$ be the joint strategy set.

If some seller's cost function were not $P_{i}$-measurable, then $C_{i}(\cdot, \omega) \neq C_{i}\left(\cdot, \omega^{\prime}\right)$ for some $\omega, \omega^{\prime} \in E \in P_{i}$. However, as sellers can observe their costs before posting prices in the market, this would mean seller $i$ could infer more information about the state of the world than their information partition provides. To rule this out, $P_{i}$-measurability will be imposed upon the cost functions.

Assumption 2.3 For every $i \in N$, the function $C_{i}(\cdot, \omega)$ is $P_{i}$-measurable.

\footnotetext{
8 To be mathematically precise, measurable with respect to the algebra generated by the elements in $P_{i}$. However, as the set $\Omega$ is finite, there is no loss of generality in using partitions to model the information restrictions.
} 
In the price choice game sellers post prices in the market and commit to meeting all the demand forthcoming at the prices posted, so there is no rationing of the market demand. As the sellers produce a perfectly homogeneous good, buyers only consume from the sellers who post the minimum price in the market. If sellers tie at the minimum price then a sharing rule determines how the market demand is split between the sellers. Two well-known rules are equal sharing and winner-takes-all sharing. Under equal sharing the market demand is split equally between sellers tieing at the minimum price, and under winner-takes-all sharing one seller is chosen at random to meet all the market demand. ${ }^{9}$ The monopoly profit of seller $i$ in state $\omega \in \Omega$ when posting price $x$ is

$$
\pi_{i}(x, \omega)=x D(x, \omega)-C_{i}(D(x, \omega), \omega),
$$

the equal shared profit when seller $i \in N$ ties at the minimum price with $r-1$, $2 \leq r \leq n$, other sellers is

$$
\pi_{i, r}^{\mathrm{ES}}(x, \omega)=\frac{1}{r} x D(x, \omega)-C_{i}\left(\frac{1}{r} D(x, \omega), \omega\right),
$$

and the winner-takes-all shared profit when seller $i \in N$ ties at the minimum price with $r-1,2 \leq r \leq n$, other sellers is

$$
\pi_{i, r}^{\mathrm{WS}}(x, \omega)=\frac{1}{r} \pi_{i}(x, \omega) .
$$

The following standard assumptions are imposed upon the monopoly and equal shared profit functions.

Assumption 2.4 For every $i \in N$ and every $\omega \in \Omega$, the functions $\pi_{i}(\cdot, \omega)$ and $\pi_{i, r}(\cdot, \omega)$ are strictly concave on $(0, \bar{x})$ and are strictly positive valued at their maximum.

One might wonder when the conditions in Assumption 2.4 are satisfied. As the cost functions are convex, the monopoly and tied profit functions will be concave with piecewise-affine (linear) market demand. More generally, as long as the market demand is sufficiently concave, Assumption 2.4 will be satisfied. Given a vector of strategies $f=\left(f_{1}, \ldots, f_{n}\right) \in L$ the payoff which seller $i$ obtains in state $\omega \in \Omega$ under equal sharing is

$$
u_{i}^{\mathrm{ES}}(f, \omega)= \begin{cases}\pi_{i}\left(f_{i}(\omega), \omega\right), & \text { if } f_{i}(\omega)<f_{j}(\omega) \text { for all } j \neq i \\ \pi_{i, r}^{\mathrm{ES}}\left(f_{i}(\omega), \omega\right), & \text { if } i \text { ties with } r-1 \text { sellers at min. price } \\ 0 & \text { if there is a } j \text { s.t. } f_{j}(\omega)<f_{i}(\omega) .\end{cases}
$$

\footnotetext{
${ }^{9}$ A broad coverage of the classical model of price competition with complete information, including recent results on the implications of different sharing rules, can be found in Baye and Kovenock (2008), and in Vives (1999, pp. 117-123).
} 
and the payoff which seller $i$ obtains in state $\omega \in \Omega$ under winner-takes-all sharing is

$$
u_{i}^{\mathrm{WS}}(f, \omega)= \begin{cases}\pi_{i}\left(f_{i}(\omega), \omega\right), & \text { if } f_{i}(\omega)<f_{j}(\omega) \text { for all } j \neq i \\ \pi_{i, r}^{\mathrm{WS}}\left(f_{i}(\omega), \omega\right), & \text { if } i \text { ties with } r-1 \text { sellers at min. price; } \\ 0 & \text { if there is a } j \text { s.t. } f_{j}(\omega)<f_{i}(\omega) .\end{cases}
$$

Let $X$ be the set of probability distributions over $\Omega$ :

$$
X=\left\{x \in \Re^{\Omega}: x(\omega) \geq 0 \text { for every } \omega \in \Omega \text { and } \sum_{\omega \in \Omega} x(\omega)=1\right\}
$$

and let $M_{i}$ be the set of probability distributions which agree with seller i's private information:

$$
M_{i}=\left\{x \in X: x(E)=\mu(E) \text { for every } E \in P_{i}\right\} .
$$

As seller $i$ cannot distinguish between different states of the world in each $E \in P_{i}$ we consider the case of maximin expected utilities (MEU) at the ex ante stage. That is, for each $E \in P_{i}$ seller $i$ considers the minimum payoff which could occur across the set of probability distributions which agrees with his private information. Therefore, given a vector of strategies $f=\left(f_{1}, \ldots, f_{n}\right) \in L$ and equal sharing the ex ante payoff of seller $i$ is:

$$
U_{i}(f)=\min _{x \in M_{i}}\left[\sum_{\omega \in \Omega} x(\omega) u_{i}^{\mathrm{ES}}(f, \omega)\right]
$$

This expression for ex ante payoff is equivalent to:

$$
U_{i}(f)=\sum_{E \in P_{i}} \mu(E)\left[\min _{\omega \in E} u_{i}^{\mathrm{ES}}(f, \omega)\right]
$$

If one replaces $u_{i}^{\mathrm{ES}}(f, \omega)$ with $u_{i}^{\mathrm{WS}}(f, \omega)$ then one obtains the expressions for ex ante payoff in the price game with winner-takes-all sharing at price ties.

Remark 2.1 The expressions for ex ante payoff are the same as those used in de Castro and Yannelis (2011) and He and Yannelis (2015a). As is well-known, these preferences are able to explain the Ellsberg (1961) violations of standard expected utility theory. If the information partition of a seller contains only singletons then the maximin expected utility coincides with standard Bayesian utilities in which seller $i$ can assign a probability to each state of the world. However, if $\omega, \omega^{\prime} \in E$ for some $E \in P_{i}$, and seller $i$ cannot distinguish between states $\omega$ and $\omega^{\prime}$ it is not unreasonable to assume that they consider the minimum utility consistent with their private information. 
A vector of strategies $f=\left(f_{1}, \ldots, f_{n}\right) \in L$ is a pure strategy price equilibrium if, for every $i \in N$,

$$
U_{i}(f) \geq U_{i}\left(f_{i}^{\prime}, f_{-i}\right) \text { for every } f_{i}^{\prime} \in L_{i}
$$

In other words, a vector of strategies $f=\left(f_{1}, \ldots, f_{n}\right) \in L$ is a pure strategy price equilibrium if it is a Nash equilibrium in the game with ex ante payoffs $U(\cdot)$. The price game with asymmetric information can be summarized by $G=$ $\left(N, \Omega,\left(C_{i}, P_{i}\right)_{i \in N}, D, \mu\right)$.

Throughout the rest of the paper it shall be assumed that all elements of the price game with asymmetric information $G=\left(N, \Omega,\left(C_{i}, P_{i}\right)_{i \in N}, D, \mu\right)$ are common knowledge amongst the sellers, except for the prior $\mu$ over $\Omega$. As a consequence, even though seller $i$ does not know the individual probabilities assigned to different states within each $E \in P_{i}$, he may know that other sellers do know which probabilities are assigned to the individual states, depending upon the specifics of the information partitions of the other sellers.

In a price game with asymmetric information, $G$, two sellers $i, j \in N$ will be said to be of the same information type if $P_{i}=P_{j}$. That is, sellers $i$ and $j$ are the same information type if their information partitions are the same. For any game $G$ let $T^{G}$ be the unique partition of the player set $N$ with the two properties: (i) if $T \in T^{G}$ and $i, j \in T$ then $i$ and $j$ are of the same information type (ii) if $T, T^{\prime} \in T^{G}$ and $i \in T$, $j \in T^{\prime}$ then $i$ and $j$ are not of the same information type.

Let $x_{B \omega}$ be the unique positive real number satisfying $\pi_{i}\left(x_{B \omega}, \omega\right)=0$ and $D\left(x_{B \omega}, \omega\right)>0$. The number $x_{B \omega}$ is the monopoly break-even price of seller $i$ in state $\omega$. The following lemma states some useful properties of the payoffs under equal sharing which are proved in Dastidar $(1995,2011)$, and discussed in detail in Vives (1999, pp. 120-122).

Lemma 2.1 Fix a price game with asymmetric information $G=\left(N, \Omega,\left(C_{i}, P_{i}\right)_{i \in N}\right.$, $D, \mu)$. Suppose $C_{i}(\cdot, \omega)=C_{j}(\cdot, \omega)$ for every $i, j \in N$ in state $\omega \in \Omega$. There exists a unique real number $x_{* \omega} \leq x_{B \omega}$ such that:

(i) $\pi_{i, r}^{\mathrm{ES}}(x, \omega) \geq \pi_{i}(x, \omega)$ for every $x \in\left[x_{* \omega}, x_{B \omega}\right]$ and every $2 \leq r \leq n$

(ii) $\pi_{i, r}^{\mathrm{ES}}(x, \omega) \geq 0$ for every $x \in\left[x_{* \omega}, x_{B \omega}\right]$ and every $2 \leq r \leq n$.

The interval in Lemma 2.1 is a subset of the Bertrand equilibrium set of the price game with two sellers. As is demonstrated in Dastidar (1995), with symmetric sellers the monopoly break-even price is always an element of the Bertrand equilibrium set. The lower bound of the equilibrium set decreases with the number of sellers. Therefore, if one considers the interval with the lower bound given by the lower bound of the twoseller Bertrand equilibrium set, and the upper bound being the monopoly break-even price, this set has all the properties described in Lemma 2.1. ${ }^{10}$

\footnotetext{
10 To see a specific interval, consider the following example from Vives (1999, p. 122). The market demand is $D(x)=\max \{0, a-b x\}$ and the cost functions of the sellers are $C(x)=c x^{2} / 2$. The monopoly break-even price is $x_{B}=a c /(2+b c)$, and with $n$ sellers the lower bound on the Bertrand equilibrium set is $a c /(2 n+b c)$. Setting $n=2$ in the lower bound one obtains the interval in Lemma 2.1 to be $[a c / 4+b c, a c / 2+b c]$.
} 


\section{The results}

In this section two important conditions are introduced. The next subsection demonstrates that if either of these conditions is relaxed then a pure strategy price equilibrium may fail to exist. The following subsection presents the main results of the paper which show that if a game satisfies both of the conditions then the game possesses a pure strategy price equilibrium.

\subsection{Two indispensable conditions for existence of pure strategy price equilibrium}

Consider a price game with asymmetric information $G=\left(N, \Omega,\left(C_{i}, P_{i}\right)_{i \in N}, D, \mu\right)$. Condition (i) requires that for every $T \in T^{G},|T| \geq 2$. The intuition behind the condition is that no seller has a monopoly over their information. Condition (ii) requires that $C_{i}(\cdot, \omega)=C_{j}(\cdot, \omega)$ for every $i, j \in N$ and every $\omega \in \Omega$. The intuition behind this condition is that the sellers have access to a common production function which generates their symmetric cost functions.

\subsubsection{A game satisfying (i) but not (ii)}

Consider a game with two sellers, $N=\{1,2\}$, and two states of the world, $\Omega=$ $\left\{\omega_{1}, \omega_{2}\right\}$. The probability distribution $\mu$ is $\mu\left(\omega_{1}\right)=\mu\left(\omega_{2}\right)=1 / 2$. The information partitions of the sellers are:

$$
P_{1}=P_{2}\left\{\left\{\omega_{1}\right\},\left\{\omega_{2}\right\}\right\} \text { for } i=1,2 \text {. }
$$

The state-contingent market demands are $D\left(x, \omega_{1}\right)=\max \{0,10-x\}$ and $D\left(x, \omega_{2}\right)=$ $\max \{0,15-x\}$. The state-contingent cost functions of the sellers are $C_{1}(x, \omega)=x$ for every $\omega \in \Omega$, and $C_{2}(x, \omega)=2 x$ for every $\omega \in \Omega$.

Clearly, this game satisfies (i) but not (ii). To see that this game fails to possess a pure strategy price equilibrium, under either equal sharing or winner-takes-all sharing, note that seller 2 will always set $f_{2}(\{\omega\})=2$ for every $\omega \in \Omega$ in any equilibrium. However, seller 1 would then prefer to post $f_{1}(\{\omega\})=2-\epsilon, \epsilon>0$. As seller 1's best response is not defined, the game fails to possess a pure strategy equilibrium.

\subsection{A game satisfying (ii) but not (i)}

Consider a game with two sellers, $N=\{1,2\}$, and two states of the world, $\Omega=$ $\left\{\omega_{1}, \omega_{2}\right\}$. The probability distribution $\mu$ is $\mu\left(\omega_{1}\right)=\mu\left(\omega_{2}\right)=1 / 2$. The information partitions of the sellers are:

$$
P_{i}=\left\{\left\{\omega_{1}\right\},\left\{\omega_{2}\right\}\right\} \text { for } i=1
$$

and 


$$
P_{i}=\{\Omega\} \text { for } i=2
$$

The state-contingent market demands are $D\left(x, \omega_{1}\right)=\max \left\{0,4 \frac{1}{20}-\frac{1}{8} x\right\}$ and $D\left(x, \omega_{2}\right)=\max \{0,24-x\}$. The state-contingent cost function of the sellers is $C_{i}(x, \omega)=x^{2}$ for every $\omega \in \Omega$ and every $i \in N$.

Clearly, this game satisfies (ii) but not (i). This game does not possess a pure strategy equilibrium under the equal sharing rule. To see this, first note that the monopoly price of sellers is the same in both states of the world $x^{M}=18$. Given the information partitions, seller 2's strategy is of the type:

$$
f_{2}\left(\left\{\omega_{1}\right\}\right)=f_{2}\left(\left\{\omega_{2}\right\}\right)=y .
$$

Seller 2 would never set $y<8$ because this is the lower bound on tied profit being non-negative in state $\omega_{2}$. If seller 2 chooses $y \in[8,18]$, then in state $\omega_{1}$ seller 1 would choose $f_{1}\left(\left\{\omega_{1}\right\}\right)=y-\epsilon, \epsilon>0$, and seller $1^{\prime} s$ best response is not well-defined. If seller 2 chooses $y>18$, then seller 1 would choose $f_{1}\left(\left\{\omega_{1}\right\}\right)=f_{2}\left(\left\{\omega_{2}\right\}\right)=18$. However, seller 2's best response would be to choose $y=18-\epsilon, \epsilon>0$, and consequently, 2's best response is not well-defined.

To see that the game fails to possess a pure equilibrium under the winner-takes-all sharing rule, note that seller 2 would always set

$$
f_{2}\left(\left\{\omega_{1}\right\}\right)=f_{2}\left(\left\{\omega_{2}\right\}\right)=12
$$

because this is the higher of the two monopoly break-even prices. However, in state $\omega_{1}$ seller 1 's best response would be to choose $f_{1}\left(\left\{\omega_{1}\right\}\right)=12-\epsilon, \epsilon>0$, and consequently, seller 1's best response is not well-defined.

\subsection{Existence of equilibrium}

The following results show that the two conditions previously discussed guarantee the existence of a pure strategy price equilibrium under equal-sharing and winner-takes-all sharing.

Proposition 3.1 Fix a price game with asymmetric information $G=\left(N, \Omega,\left(C_{i}\right.\right.$, $\left.\left.P_{i}\right)_{i \in N}, D, \mu\right)$ and equal sharing at prices ties. If the following two conditions are satisfied:

(i) For every $T \in T^{G},|T| \geq 2$

(ii) $C_{i}(\cdot, \omega)=C_{j}(\cdot, \omega)$ for every $i, j \in N$ and every $\omega \in \Omega$.

Then the game $G$ possesses a pure strategy price equilibrium.

Proof Let $G=\left(N, \Omega,\left(C_{i}, P_{i}\right)_{i \in N}, D, \mu\right)$ be a game satisfying the conditions in the Proposition. We shall construct one price equilibrium of the game. The numbers $\left(x_{B \omega}\right)$ can be ordered using the complete order $\leq$. Suppose, without loss of generality, that

$$
x_{B \omega_{1}} \leq x_{B \omega_{2}} \leq \cdots \leq x_{B \omega_{m}}
$$


Let $\left(y_{\omega}\right)_{\omega \in \Omega}$ be a set of real numbers with the two properties: (i) $y_{\omega} \in\left[x_{* \omega}, x_{B \omega}\right]$ (ii) if $x_{B \omega} \leq x_{B \omega^{\prime}}$ then $y_{\omega} \leq y_{\omega^{\prime}}$.

Let the strategies of the sellers be as follows. For every $i \in N$ and $E \in P_{i}$

$$
f_{i}^{*}(E)=\max _{\omega \in E} y_{\omega}
$$

The strategies $f^{*}=\left(f_{1}^{*}, \ldots, f_{n}^{*}\right)$ are a pure strategy price equilibrium of the game $G$. To see this, note that for every $i \in N$, and every $\omega \in \Omega$, we have

$$
u_{i}^{\mathrm{ES}}\left(f^{*}, \omega\right) \geq 0
$$

because across all the states of the world $i$ either ties at the minimum price and, from Lemma 2.1, obtains non-negative profit, or $i$ does not post the minimum price and obtains zero profit. Therefore, for every $E \in P_{i}$

$$
\min _{\omega \in E} u_{i}^{\mathrm{ES}}\left(f^{*}, \omega\right) \geq 0
$$

and as $\mu(E)>0$

$$
\mu(E)\left[\min _{\omega \in E} u_{i}^{\mathrm{ES}}\left(f^{*}, \omega\right)\right] \geq 0 .
$$

Hence, $U_{i}\left(f^{*}\right) \geq 0$ for every $i \in N$.

Suppose, for some $E \in P_{i}$, seller $i$ decided to deviate to a strategy $f_{i}^{\prime}$ with $f_{i}^{\prime}(E)=$ $y^{\prime}<f_{i}^{*}(E)$. In event $E$ their payoff is

$$
\mu(E)\left[\min _{\omega \in E} u_{i}^{\mathrm{ES}}\left(f_{i}^{\prime}, f_{-i}^{*}, \omega\right)\right] .
$$

However

$$
\min _{\omega \in E} u_{i}^{\mathrm{ES}}\left(f_{i}^{\prime}, f_{-i}^{*}, \omega\right)<0
$$

because for at least one state in $E$ seller $i$ posts the unique minimum price in the market, and $y^{\prime}$ is less than the monopoly break-even price. As $\mu(E)>0$,

$$
\mu(E)\left[\min _{\omega \in E} u_{i}^{\mathrm{ES}}\left(f_{i}^{\prime}, f_{-i}^{*}, \omega\right)\right]<0 .
$$

Suppose, for some $E \in P_{i}$, seller $i$ decided to deviate to a strategy $f_{i}^{\prime}$ with $f_{i}^{\prime}(E)=$ $y^{\prime}>f_{i}^{*}(E)$. Then,

$$
\mu(E)\left[\min _{\omega \in E} u_{i}^{\mathrm{ES}}\left(f_{i}^{\prime}, f_{-i}^{*}, \omega\right)\right]=0
$$


because $|T| \geq 2$ for every $T \in T^{G}$ and $i$ never posts the minimum price in the market in any state in $E$.

Remark 3.1 If each seller's cost function is strictly convex, then $x_{* \omega}<x_{B \omega}$ for every $\omega \in \Omega$, and it follows from the proof of Proposition 3.1 that the game possesses a continuum of pure strategy price equilibria. This is an interesting analogue of the standard complete information game. Dastidar (1995) demonstrated that with symmetric strictly convex costs there is a continuum of pure strategy price equilibria. Proposition 3.1 shows that, even requiring the $P_{i}$-measurability of each seller's prices, with symmetric strictly convex costs, there may exist a continuum of pure strategy price equilibria.

Example 3.2 Consider a game with four sellers, $N=\{1,2,3,4\}$, and two states of the world, $\Omega=\left\{\omega_{1}, \omega_{2}\right\}$. The probability distribution, $\mu$, is $\mu\left(\omega_{1}\right)=\mu\left(\omega_{2}\right)=1 / 2$. There is equal sharing at price ties. The information partitions of the sellers are:

$$
P_{i}=\left\{\left\{\omega_{1}\right\},\left\{\omega_{2}\right\}\right\} \text { for } i=1,2
$$

and

$$
P_{i}=\{\Omega\} \text { for } i=3,4 \text {. }
$$

The state-contingent market demands are $D\left(x, \omega_{1}\right)=\max \{0,6-x\}$ and $D\left(x, \omega_{2}\right)=$ $\max \{0,12-x\}$. The state-contingent cost function of the sellers is $C(x, \omega)=x^{2}$ for every $\omega \in \Omega$. Given these market primitives, the intervals discussed in Lemma 2.1 are:

$$
\left[x_{* \omega_{1}}, x_{B \omega_{1}}\right]=[2,3]
$$

and

$$
\left[x_{* \omega_{2}}, x_{B \omega_{2}}\right]=[4,6] \text {. }
$$

One pure strategy price equilibrium of the game is:

$$
f_{i}^{*}\left(\left\{\omega_{1}\right\}\right)=3 \text { and } f_{i}^{*}\left(\left\{\omega_{2}\right\}\right)=4 \text { for } i=1,2
$$

and

$$
f_{i}^{*}\left(\left\{\omega_{1}\right\}\right)=f_{i}^{*}\left(\left\{\omega_{2}\right\}\right)=4 \text { for } i=3,4
$$

Proposition 3.3 Fix a price game with asymmetric information $G=\left(N, \Omega,\left(C_{i}\right.\right.$, $\left.\left.P_{i}\right)_{i \in N}, D, \mu\right)$ and winner-takes-all sharing at prices ties. If the following two conditions are satisfied:

(i) For every $T \in T^{G},|T| \geq 2$

(ii) $C_{i}(\cdot, \omega)=C_{j}(\cdot, \omega)$ for every $i, j \in N$ and every $\omega \in \Omega$. 
Then the game $G$ possesses a pure strategy price equilibrium.

Proof Let $G=\left(N, \Omega,\left(C_{i}, P_{i}\right)_{i \in N}, D, \mu\right)$ be a game satisfying the conditions in the Proposition. Consider the numbers $\left(x_{B \omega}\right)_{\omega \in \Omega}$.

Define the strategies of the sellers be as follows. For every $i \in N$ and $E \in P_{i}$,

$$
f_{i}^{*}(E)=\max _{\omega \in E} x_{B \omega}
$$

The strategies $f^{*}=\left(f_{1}^{*}, \ldots, f_{n}^{*}\right)$ are a pure strategy price equilibrium of the game $G$. To see this, note that for every $i \in N$ and $E \in P_{i}$,

$$
\mu(E)\left[\min _{\omega \in E} u_{i}^{\mathrm{WS}}\left(f^{*}, \omega\right)\right]=0 .
$$

because for every $i \in N$ and $\omega \in \Omega$ each seller either does not post the minimum price in the market, or ties at the monopoly break-even price. Therefore, $U_{i}\left(f^{*}\right)=0$ for every $i \in N$.

Suppose, for some $E \in P_{i}$, seller $i$ decided to deviate to a strategy $f_{i}^{\prime}$ with $f_{i}^{\prime}(E)=$ $y^{\prime}<f_{i}^{*}(E)$. Then,

$$
\mu(E)\left[\min _{\omega \in E} u_{i}^{\mathrm{WS}}\left(f_{i}^{\prime}, f_{-i}^{*}, \omega\right)\right]<0
$$

because the minimum across the states in $E$ includes one state when $i$ posts the unique minimum price in the market and obtains the monopoly profit. However, $y^{\prime}$ is less than the monopoly break-even price.

Suppose, for some $E \in P_{i}$, seller $i$ decided to deviate to a strategy $f_{i}^{\prime}$ with $f_{i}^{\prime}(E)=$ $y^{\prime}>f_{i}^{*}(E)$. Then,

$$
\mu(E)\left[\min _{\omega \in E} u_{i}^{\mathrm{WS}}\left(f_{i}^{\prime}, f_{-i}^{*}, \omega\right)\right]=0
$$

because $|T| \geq 2$ for every $T \in T^{G}$, and consequently, $i$ does not post the minimum price in the market across the states in $E$ and obtains zero profit.

Remark 3.2 The result in Proposition 3.3 still holds even of one dispenses with the assumption of convex costs imposed in Assumption 2.1. This is because under the winner-takes-all sharing rule the payoffs are determined by the monopoly profit function. Dastidar (2011) has demonstrated that with symmetric sub-additive cost functions (which generalizes the concept of concave costs), and equal sharing at price ties, there does not exist a pure or mixed strategy price equilibrium. However, under the winnertakes-all sharing rule and decreasing returns to scale costs, Baye and Morgan (2002), showed that a pure strategy price equilibrium may exist (even with certain types of discontinuities in the profit function). 
Example 3.4 Consider a market with four sellers, $N=\{1,2,3,4\}$, and three states of the world, $\Omega=\left\{\omega_{1}, \omega_{2}, \omega_{3}\right\}$. The probability distribution, $\mu$, is $\mu\left(\omega_{1}\right)=\mu\left(\omega_{2}\right)=$ $\mu\left(\omega_{3}\right)=1 / 3$. The winner-takes-all sharing rule is used at price ties. The information partitions of the sellers are:

$$
P_{i}=\left\{\left\{\omega_{1}, \omega_{2}\right\},\left\{\omega_{3}\right\}\right\} \text { for } i=1,2
$$

and

$$
P_{i}=\left\{\left\{\omega_{1}\right\},\left\{\omega_{2}, \omega_{3}\right\}\right\} \text { for } i=3,4
$$

The state-contingent market demands are $D\left(x, \omega_{1}\right)=\max \{0,4-x\}, D\left(x, \omega_{2}\right)=$ $\max \{0,8-x\}$ and $D\left(x, \omega_{3}\right)=\max \{0,12-x\}$. The state-contingent cost function of the sellers is $C(x, \omega)=x^{2}$ for every $\omega \in \Omega$. Given these market primitives, the monopoly break-even prices are $x_{B \omega_{1}}=2, x_{B \omega_{2}}=4$ and $x_{B \omega_{3}}=6$.

A pure strategy price equilibrium of the game is:

$$
f_{i}^{*}\left(\left\{\omega_{1}, \omega_{2}\right\}\right)=4 \text { and } f_{i}^{*}\left(\left\{\omega_{3}\right\}\right)=6 \text { for } i=1,2
$$

and

$$
f_{i}^{*}\left(\left\{\omega_{1}\right\}\right)=2 \text { and } f_{i}^{*}\left(\left\{\omega_{2}, \omega_{3}\right\}\right)=6 \text { for } i=3,4
$$

\section{Concluding remarks}

This work has analyzed a price choice game in a homogeneous-good market in which the sellers may have asymmetric information about the state of the world and have maximin expected utilities. Unlike other models which have studied price competition under symmetric uncertainty, the model presented here permits general asymmetries of information of the kind usually studied in the context of general equilibrium models. The results indicate that if the sellers have symmetric costs, and there are at least two sellers of each information type then there exists a pure strategy price equilibrium under both equal sharing and winner-takes-all sharing at price ties. In many games with discontinuous payoffs it is often possible to prove that an equilibrium exists, but finding specific examples of such equilibria can be difficult. In contrast, the existence proofs presented here are both direct and constructive. This should help make it possible to study the equilibria of the model under different extensions. To conclude, two possible extensions of the model, which could yield further interesting results, are presented below.

- If sellers have strictly convex costs, and the game satisfies the conditions of Proposition 3.1, then there exists a continuum of pure strategy price equilibria (see Remark 3.1). It would be interesting to know whether some of these equilibria are more plausible than others. One refinement of the Nash equilibrium which has been applied to price games is the coalition-proof refinement of 
Bernheim et al. (1987). This refinement is appropriate in games where the players can communicate with each other but cannot write binding agreements. In Chowdhury and Sengupta (2004) it was shown that Bertrand games possess a coalition-proof equilibrium under quite general conditions. Whether the asymmetric information game studied here possesses a coalition-proof equilibrium is an interesting open question. ${ }^{11}$ More recently, Andersson et al. (2014) have also proposed a Nash equilibrium refinement based upon strategic uncertainty. They applied this refinement to standard complete information price games and showed that it selected a unique price equilibrium- different from the coalition-proof equilibrium. This alternative refinement could also be applied to the asymmetric information game.

- Throughout the paper it has been assumed that sellers know their costs before they posted prices in the market. This was why the assumption of $P_{i}$-measurability was imposed upon the cost functions. However, in many real-world scenarios, sellers have to post prices without being fully aware of their costs. In Wambach (1999) it was assumed that sellers were uncertain of their costs and were also risk-averse. A consequence of this is that, even with constant marginal costs, the usual Bertrand paradox fails to hold, with prices being posted above marginal cost-even as the number of sellers becomes large. It would be interesting to extend the asymmetric information game studied here to consider the possibility that sellers are uncertain about their costs when posting prices in the market.

Open Access This article is distributed under the terms of the Creative Commons Attribution 4.0 International License (http://creativecommons.org/licenses/by/4.0/), which permits unrestricted use, distribution, and reproduction in any medium, provided you give appropriate credit to the original author(s) and the source, provide a link to the Creative Commons license, and indicate if changes were made.

\section{References}

Allingham, M.: Choice Theory: A Very Short Introduction. Oxford University Press, New York (2002)

Andersson, O., Argenton, C., Weibull, J.: Robustness to strategic uncertainty. Games Econ. Behav. 85, 272-288 (2014)

Bagh, A.: Pure strategy equilibria in bertrand games with discontinuous demand and asymmetric tiebreaking rules. Econ. Lett. 108, 277-279 (2010)

Baye, M.R., Kovenock, D.: Bertrand Competition. In: Durlauf, S.N., Blume, L.E. (eds.) New Palgrave Dictionary of Economics. Palgrave Macmillan, Basingstoke (2008)

Baye, M., Morgan, J.: Winner-take-all price competition. Econ. Theory 19, 271-282 (2002)

Bernheim, D., Peleg, B., Whinston, M.: Coalition-proof nash equilibria I: concepts. J. Econ. Theory 42, $1-12$ (1987)

Blume, A.: Bertrand without fudge. Econ. Lett. 78(2), 167-168 (2003)

de Castro, L.I., Yannelis, N.C.: Ambiguity aversion solves the conflict between efficiency and incentive compatibility. University of Manchester Discussion Paper (2011)

Chowdhury, P., Sengupta, K.: Coalition-proof bertrand equilibria. Econ. Theory 24, 307-324 (2004)

Correia-da-Silva, J., Hervés-Beloso, C.: Prudent expectations equilibrium in an economy with uncertain delivery. Econ. Theory 39, 67-92 (2009)

\footnotetext{
11 Extending coalition-proof refinements to asymmetric information settings is quite a subtle issue because one can permit the possibility that sellers share information via communication systems of the type studied in Wilson (1978), but self-enforcing conditions also need to be imposed upon the information sharing.
} 
Dastidar, K.: On the existence of pure strategy bertrand equilibrium. Econ. Theory 5, 19-32 (1995)

Dastidar, K.: Existence of bertrand equilibrium revisited. Int. J. Econ. Theory 7, 331-350 (2011)

Debreu, G., Scarf, H.: A limit theorem on the core of an economy. Int. Econ. Rev. 4, 235-246 (1963)

Ellsberg, D.: Risk, ambiguity and the savage axioms. Q. J. Econ. 75, 643-669 (1961)

Gilboa, I., Schmeidler, D.: Maximin expected utility with non uniqiue prior. J. Math. Econ. 18, 141-153 (1989)

He, W., Yannelis, N.C.: Equilibrium theory under ambiguity. J. Math. Econ. 61, 86-95 (2015a)

He, W., Yannelis, N.C.: Discontinuous games with asymmetric information: an extension of reny's existence theorem. Games Econ. Behav. 91, 26-35 (2015b)

He, W., Yannelis, N.C.: Existence of equilibria in discontinuous bayesian games. J. Econ. Theory 162, 181-194 (2016)

Hoernig, S.: Bertrand games and sharing rules. Econ. Theory 31, 573-585 (2007)

Reny, P.: On the existence of pure and mixed strategy nash equilibria in discontinuous games. Econometrica 67, 1029-1056 (1999)

Saporiti, A., Coloma, G.: Bertrand competition in markets with fixed costs. BE J. Theor. Econ. 10(1), Article 27 (2010)

Spulber, D.: Bertrand competition when rivals' costs are unknown. J. Ind. Econ. 43, 1-11 (1995)

Vives, X.: Oligopoly Pricing: Old Ideas and New Tools. MIT Press, Cambridge (1999)

Wambach, A.: Bertrand competition under cost uncertainty. Int. J. Ind. Organ. 17, 941-951 (1999)

Wilson, R.: Information, efficiency and the core of an economy. Econometrica 4, 807-816 (1978) 